\title{
Recent Developments in the Measurement of Low Particulate Emissions from Mobile Sources: A Review of Particle Number Legislations
}

\author{
Oliver F. Bischof ${ }^{1}$
}

Received: 16 February 2015 /Revised: 12 April 2015 /Accepted: 14 April 2015 /Published online: 28 April 2015

(C) Springer SIP, AG 2015

\begin{abstract}
The combination of modern engine technologies, efficient aftertreatment, and tighter emission standards resulted in drastically lower particulate emission levels in Europe today. The conventional methods defined to test particulate emissions fail at such low levels as they are aimed at determining the mass of the particles collected on a filter (PM). This led to particle number ( $\mathrm{PN}$ ) measurement having become established as the method of choice to test for low particulate emissions from mobile sources. At present, there are several emission legislations that have added PN as an additional emission parameter, while there are no mandatory regulations for ambient air monitoring anywhere in the world so far. An overview of already enforced as well as an outlook of upcoming emission legislations for mobile sources that will require PN measurement will be given in this review and corresponding test solutions are described. Special emphasis is given to emission testing of construction machinery diesel particle filters in the field. Portable test equipment capable of measuring nonvolatile PN from such combustion sources is explained. Despite the progress, challenges in the measurement of low particle emissions remain, including the fact that solid particles that exist in the sub-23-nm-size range could be of more importance than originally thought.
\end{abstract}

Keywords Particulate emissions · Particle number legislation $\cdot$ Diesel particle filter $\cdot$ Solid-particle number

Oliver F. Bischof

obischof@tsi.com

1 TSI GmbH, Particle Instrument, Neuköllner Strasse 4, 52068 Aachen, Germany

\section{Introduction}

During the past few years, several emission legislations have been amended and new regulations have been introduced. In consequence, more stringent levels for particulate emissions from light-duty and heavy-duty vehicles have been imposed, with additional regulations for off-road machinery and aircraft engines under preparation. For example, the Euro VI standard for heavy-duty diesel engines that became effective for new type approvals in 2013 resulted in a $90 \%$ reduction of particulate mass compared to the Euro III standard that came into effect only 13 years earlier. When considering particle number, that decreases amounts even to $99.8 \%$. These tighter emission standards in combination with state-of-the art engine technologies and very efficient emission control devices such as diesel particulate filters (DPF) and gasoline particulate filters (GPF) have resulted in the drastically lower particulate emission levels.

As a result of the lower emission levels, conventional test methods to determine and certify particulate emissions have reached their measurement limits. A few years ago, particulate matter (PM) was the only parameter of the particulate emissions that was measured. It is the PM mass test methods that regularly fail at the low emission levels seen today. During filter measurements, the mass of particles collected on the filter is only a tiny fraction of the overall filter mass, typically just three orders of magnitude of the total mass. This puts the microbalances and ultra-microbalances used to weigh such filters at the limit of their detection as weighing anything close to the weight of a blank filter is extremely challenging. An added complication with that method is that the volatile fraction of the material collected on the filter can be many times the mass of solid particles collected, and that different filter materials have a different response to such volatiles. Established real-time PM methods do not fare better and fail 
at low emission levels as well, including those used for legal certification of engines. Schlatter [26] and Spiegel et al. [31] reported that transmission smoke meters and opacimeters reach the limit of their capabilities as the measurement principles become less sensitive at smaller particle sizes and low concentrations. They concluded that opacimeters are not suitable anymore for the current generation of diesel engines that meet the latest Euro 5 and 6 standards.

In addition, PM mass is a measurement parameter that is ill-suited to begin with for the testing of efficient diesel particle filters (DPF) as they are nowadays commonly used to clean the emission of passenger cars, trucks, and buses. The reason lies in the fact that PM mass is essentially insensitive to the fill state of a DPF or to its porosity. PM mass emission tests also do not necessarily permit distinguishing between a DPF that is working properly from one that is malfunctioning or even disabled by the user. Instead, the test method should be sensitive enough to also detect a DPF that fails in the field, e.g., due to cracking, melting, or a malfunctioning regeneration system. Such malfunctioning DPFs might still capture the majority of the particulate mass but could permit a high number of particles to pass through at the same time, which is a failure that is very hard for conventional mass- and opacitybased test systems to detect.

The solution to this measurement predicament is the addition of the particle number $(\mathrm{PN})$ parameter. Real-time measurements of the particle number and size distribution of engine exhaust emissions have been made since the early 1970s [30]. The measurement of nonvolatile $\mathrm{PN}$ is superior to the gravimetric PM mass method both in terms of its precision and speed, but it is also more robust overall. The PN measurement method has been shown to be three orders of magnitude more sensitive than PM measurements are and has a substantially better signal-to-noise ratio, in particular for low particle emissions [11]. Particle number measurements can be accomplished with relative ease; in particular as standardized emissions test systems are now readily available. Furthermore, the PN method offers the added benefit that it can be used to estimate health impacts of the ultrafine particle fraction, which is the largest contributor to the particle number concentration [20]. Exposure to ultrafine particles is an important issue in particular in urban areas and near highly frequented roads. The combined benefits of the solid PN measurement method led to new emission legislations for particle number being considered and subsequently implemented.

\section{Existing Emission Legislations for Particle Number}

\subsection{Light-Duty Diesel Legislation}

Switzerland played the pioneering role in the establishment of emission standards for particle number concentration in
Europe. In the mid 1990s, the VERT consortium (Verminderung der Emissionen von Realmaschinen im Tunnelbau) was formed with the aim to achieve a drastic reduction of soot emitted by machinery used in tunnel construction. VERT comprehensively showed that particulate trap technology, and DPFs, in particular, were the only acceptable choice to efficiently remove nanometer-sized particles from the exhaust gas. The list of available filters for diesel engines that was first put together by VERT in 1998 mandated $95 \%$ filtration efficiency for solid particles in the size range from 20 to $500 \mathrm{~nm}$, which was likely the first establishment of a particle number count criterion for exhaust emissions. Using that requirement, VERT demonstrated filtration efficiencies for ultrafine particles of more than $99 \%$ by their number count [18].

In 2001, the Particulate Measurement Programme (PMP) was formed as a collaborative program lead by the UK's Department for Transport under the auspices of the United Nation's Working Party on Pollution and Energy (UNECE GRPE), with stakeholders from a number of European Union countries as well as Switzerland. The PMP working group formulated a three-phased approach. In phase 1 , various measurement systems capable of measuring several key particle parameters and properties were evaluated. An important milestone was the comparison study of particle measurement systems for future type approval at EMPA in Switzerland during PMP phase 2, which resulted in a noteworthy final report with far-reaching consequences [19]. Among other conclusions, specific recommendations were made to complement an improved PM filter measurement method by one of solid particle number counts. Phase 3 of that program then validated the two recommended measurement techniques in interlaboratory test programs. On a European Union level, UK experts proposed a revised $\mathrm{PM}$ procedure as well as the introduction of particle number count to GRPE in June 2007, a proposal that was not adopted at first. The formal vote in favor of that proposal took place 1 year later, and the amendments to UNECE Regulation No. 83 entered into force with the official publication of the procedures during February 2009. The new annex for Regulation 83 integrated modifications to the PM measurement procedure and effectively introduced the particle number procedure for certification testing. Since September 2011, the Euro 5B standard requires a PN limit of $6 \times 10^{11}$ particles $/ \mathrm{km}$ for type approval of all new light-duty diesel vehicles, the first PN legislation implemented by the European Union. The test cycle that has to be used is the New European Driving Cycle (NEDC), which consists of an urban cycle that is run four times and an extra-urban cycle. The complete emissions test procedure for the PN measurement is described in detail in Regulation No. 83 [22]. 


\subsubsection{Particulate Measurement Programme Method}

The test procedure for solid particle number in Regulation No. 83 is based on the Particulate Measurement Programme (PMP) method and is explained in great detail elsewhere [6, 10]. PMP specifies that only solid particles must be measured. Semi-volatile and volatile particles that are formed by nucleation of gaseous components as the exhaust cools were to be excluded from the measurement as they can contribute significantly to variability. While the terminology solid particle is not always used consistently, it is aimed at material that does not evaporate at temperatures of $300{ }^{\circ} \mathrm{C}$, so a more correct designation would be nonvolatile particles. It should be pointed out that the definition of solid as insoluble or nonvolatile is also important in an occupational health context. The German Social Accident Insurance used it since 1996 for particulate materials in the MAK limit values definition for A-dust and Edust (TRGS 900) [33]. While the primary method of eliminating volatile particles is thermodilution, the PMP measurement system incorporates a secondary safety factor as the smallest particle size detection limit of the particle number counter is adjusted to $23 \mathrm{~nm}$.

In essence, the PMP method consists of the following steps shown in Fig. 1. Vehicle exhaust is first diluted with HEPAfiltered air in a constant volume sampling (CVS), full flow dilution tunnel. The exhaust gas is then extracted from the CVS tunnel through a sampling system that includes a particle pre-separator for the removal of the few coarse particles larger than $2.5 \mu \mathrm{m}$ that are not measured by this method and an exhaust particle transfer system. Subsequently, the exhaust gas sample is diluted with heated air by a factor of at least ten in a primary dilution stage. This preconditioning is done at temperatures larger than $150{ }^{\circ} \mathrm{C}$ in order to avoid condensation of raw exhaust gas compounds that are included in the sample. From the primary diluter, the sample passes through the evaporation tube that is externally heated up to $400{ }^{\circ} \mathrm{C}$ before it enters a secondary dilution stage, where all volatile particles are removed from the vehicle exhaust and only solid particles remain. Finally, the sample enters the particle number counting device. The PMP method always employs a full flow condensation particle counter (CPC; [29]) for that purpose. It has to meet a required counting accuracy of $\pm 10 \%$ across the range from 1 to 10,000 particles $/ \mathrm{cm}^{3}$ with a $50 \%$ counting efficiency at a particle size of $23 \mathrm{~nm}$. The most commonly used condensation particle counters are based on the model 3790 Engine Exhaust CPC (TSI Inc., St. Paul, MN, USA; [23]). The use of CPCs raised the question of a traceable method for their calibration, in particular, with respect to their smallest particle size detection limit, counting efficiency, and concentration linearity. This subject has been addressed at length elsewhere $[5,9,15]$.

\subsubsection{Sub-23-nm Particles}

The PMP method defines that only solid particles above a 23nm threshold shall be measured. This particle size was considered to be larger than the size of typical volatile nucleation mode particles but smaller than the main accumulation mode of solid diesel particles. The motivation was to exclude volatile particles from the number concentration measurement to increase the stability and repeatability of the measurement method, which evidently proved to be the case. The threshold

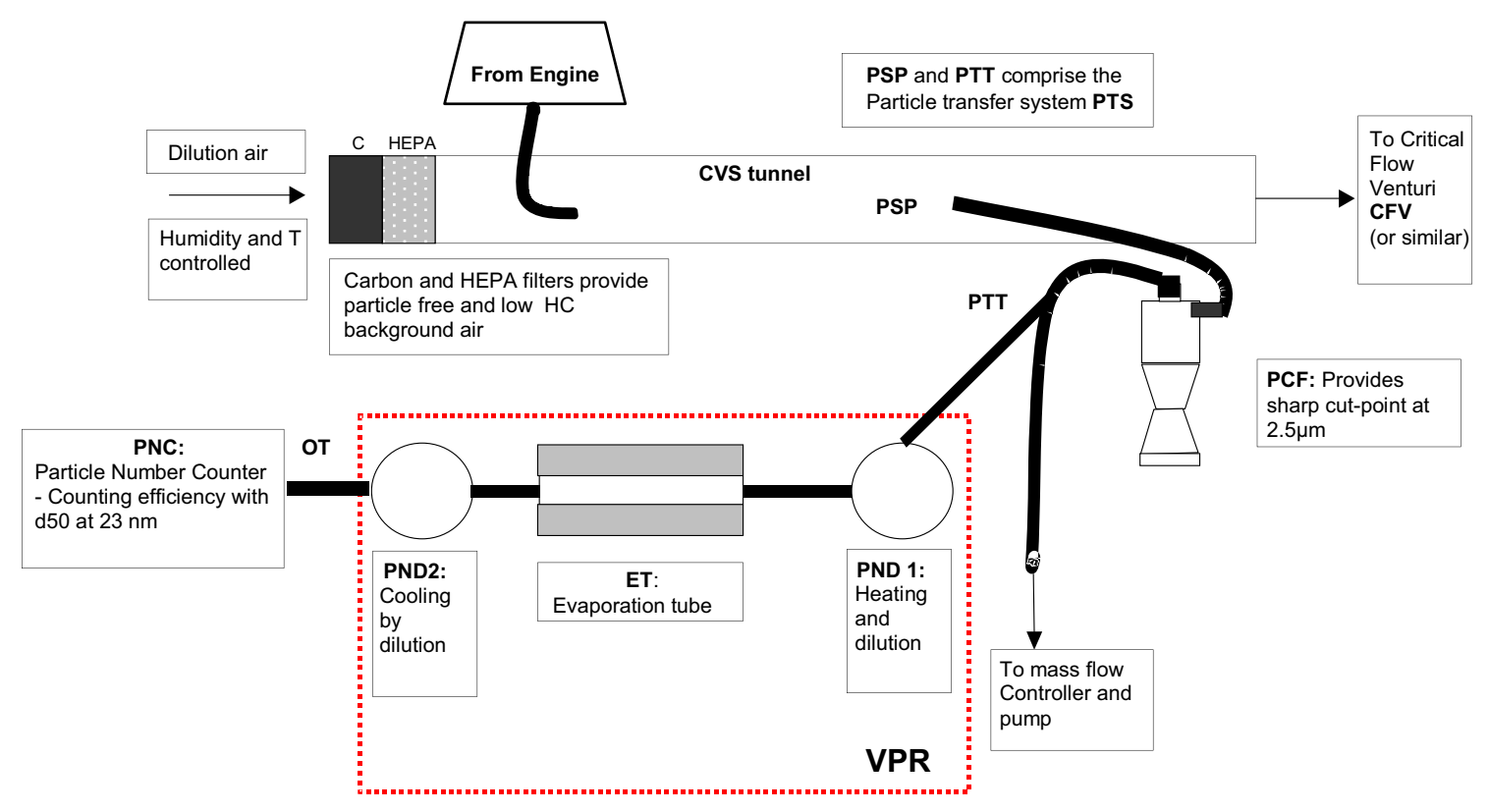

Fig. 1 Schematic of the measurement set-up following the PMP method (UNECE Regulation No. 83) 
was chosen rather arbitrarily during the early stages of the program as it could be easily implemented in then existing CPC instruments by varying the temperature difference required for the activation of the particles to be measured. Since then, there has been much discussion about the validity and usefulness of a lower size limit at exactly $23 \mathrm{~nm}$. Giechaskiel and Martini [7, 8] showed that sub-23-nm particles can be found in emissions from diesel engines without exhaust aftertreatment at low loads. Processes like the renucleation of sulfuric acid and semi-volatile material result in the formation of volatile particles in the lower nanometer range. Several studies have also shown the existence of solid particles in vehicle emissions below that threshold [12, 17, 37]. For instance, the regeneration of DPFs in modern vehicles can produce an emission of solid particles that is less than $23 \mathrm{~nm}$ in size. In 2012, Kittelson presented his findings that metal-based additives in oil and fuel can generate large numbers at small particle sizes [13]. Further work may lead to a revision of the 23-nm cutoff size as, from a measurement perspective, it is already possible to reduce the lower limit to $10 \mathrm{~nm}$ with existing CPC instruments.

\subsubsection{Turn-Key Test Systems}

The first solid particle counter, the reference "Golden" particle measurement system used during the interlaboratory test program of PMP's phase 3 from 2004 to 2006 [2, 6] was provided by Matter Engineering AG (Wohlen, Switzerland). Matter Engineering holds a patent that describes the dilution and heating of the exhaust gas sample to suppress volatile compounds before a subsequent measurement by a suitable detector (US 7,682,426), which is the exact process that was implemented by PMP [34]. The Golden instrument in essence consisted of individual components such as a rotating disk diluter with evaporation tube (Matter Engineering AG), a CPC 3010D (TSI Inc.), and a control unit mounted in a 19in. mobile rack.

Since that time, dedicated solid particle counting test systems have been introduced. They incorporate everything needed to perform PMP-compliant measurements for Euro $5 \mathrm{~b}$ and 6 certification of vehicle emissions in more compact designs that can be fully integrated into test rig automation environments. These turn-key solutions have in common that they combine the sampling line and thermodiluters, albeit of different designs, with a condensation particle counter. The most important solutions used in the automotive testing market have been realized in the AVL Particle Counter (APC 489, AVL List GmbH, Graz, Austria) and the MEXA-2000SPCS (HORIBA Ltd, Kyoto, Japan) (Fig. 2). Additional information on these PMP-systems can be found in Giechaskiel et al. [6] and Wei et al. [35]. Further test solutions that are for the most part of local significance exist in other countries, including France, Germany, China, and Japan.

\subsection{Heavy-Duty Diesel Legislation}

Following the completion of the interlaboratory test for lightduty vehicles and the corresponding report, PMP started the heavy-duty program in 2008. This Inter-Laboratory Correlation Exercise (ILCE) used the measurement approach developed in the light-duty exercise and involved a Golden heavy-duty test engine fitted with a DPF. Its validation phase was limited to four laboratories based in the European Union and one in Switzerland, although it allowed participation from additional laboratories worldwide to participate in the roundrobin and conduct their own testing if they followed the same test protocol. The Golden particle measurement systems used during the heavy-duty interlaboratory testing were two prototypes of the solid particle counting systems (SPCS) developed by HORIBA Ltd. [36]. An extensive report of the experimental work completed during this exercise has been published by Andersson et al. [3].

With the heavy-duty interlaboratory program concluded, the European Commission published a formal proposal for Euro VI emission regulations for on-road heavy-duty engines. This draft considered the implications of differences found between light- and heavy-duty testing results. It added PN limits for solid particles and followed the PMP method, with the major difference that optionally also partial flow dilution systems can be used for the type approval of new heavy-duty diesel engines. The amendments to UNECE Regulation No. 49 for heavy-duty vehicles were introduced in 2011 and became effective from January 2013 [21]. Since then, new heavy-duty diesel engines have to meet a Euro VI emissions level of $8 \times 10^{11}$ particles $/ \mathrm{kWh}$ on the World Harmonized Stationary Cycle (WHSC) and of $6 \times 10^{11}$ particles $/ \mathrm{kWh}$ on the World Harmonized Transient Cycle (WHTC).

\subsection{Gasoline DI Engine Legislation}

While the new emission legislations for particle number were only applied to diesel vehicles and engines initially, an extension that also covers vehicles using gasoline direct injection engines was subsequently addressed. This caused the amendment of Regulation No. 459, which required that all new gasoline-fueled, spark-ignition light-duty vehicles equipped with direct injection will also have to meet a PN limit in Europe. It entered into force in September 2014, and since that time, all new light-duty vehicles with gasoline direct injection engines have to meet the Euro $6 \mathrm{~b}$ emission level of $6 \times 10^{12}$ particles/ $\mathrm{km}$ tested under the New European Driving Cycle. In September 2017, the initial PN limit will be reduced further to a limit value for Euro $6 \mathrm{c}$ of $6 \times 10^{11}$ particles $/ \mathrm{km}$, which corresponds to the PN limit value for light-duty diesel vehicles. The measurement method once again follows the PMP, so the same test procedures and type of solid particle counting test systems can be used. It should be noted that this legislation 
Fig. 2 Commercially available instruments for PMP-compliant testing: AVL Particle Counter (APC) 489 shown on the left, HORIBA MEXA-2000SPCS on the right (photos $(\mathcal{)}$ AVL List GmbH; HORIBA, Ltd)

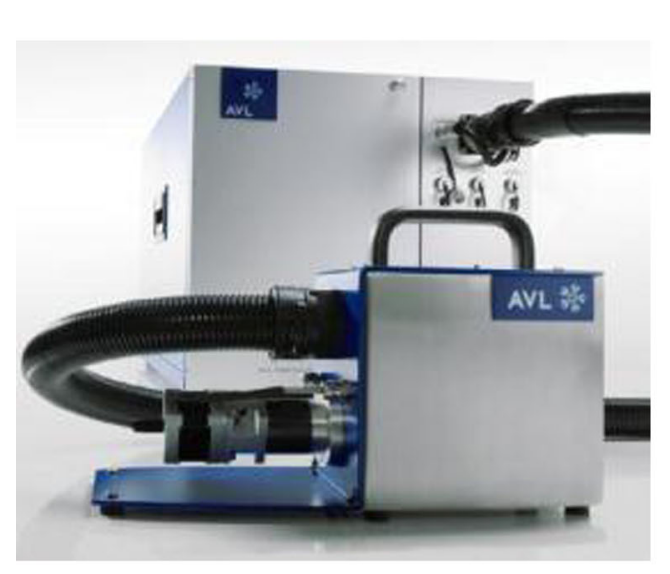

does not apply to port-fuel injection (PFI) engines, and that there is currently no regulation being planned that would extend the PN emissions level to such vehicles.

\section{Pending Emission Legislations for Particle Number}

\subsection{Off-Road Construction Machinery, Swiss Legislation}

The VERT project, and later the ongoing activities of the VERT Association, continued to drive the demand of applying best available technology to the reduction of emissions from construction machines with diesel engines and the use of particle number measurement for the quantification of these efforts. In June 2012, Switzerland adopted another pioneering role by announcing the intent to introduce a new legislation on portable particle counters for the periodic control of off-road construction machinery [27]. The Swiss Ordinance of Air Pollution Control (OAPC), a law enacted by the Swiss Government, is the first legislation worldwide that effectively limits PN emissions of off-road vehicles for machine homologation and periodic on-site emission control. This legislation effectively takes PN emissions testing out of test bench environments and into the field. High demands are made on the measuring instruments through the requirement that the machinery can only be operated when fitted with a DPF system which meets the requirements specified in OAPC or that it does not exceed a PN limit value of $10^{12}$ particles $/ \mathrm{kWh}$. Up to now, biannual tests of the particulate emission have to be conducted with opacimeters. Due to the requirement of construction machines in Switzerland to be equipped with DPFs and because of the specific new requirements for nanoparticle measurements, opacimeters will once more need to be replaced by a test system that is more sensitive to low particulate emissions.

The amendment of the Ordinance on Exhaust Gas Analysers (VAMV) with nanoparticle measurement instruments has been in place since January 1, 2013. Regulation SR 941.242 mandates the in-use compliance testing of all construction machinery DPFs, which is typically done in the field. The regulation is relevant for a wide array of products as diverse as for instance excavators, bulldozers, front loaders, fork-lifts, and power generators. The PN regulation has been published on January 1, 2015 and is expected to be promulgated within a few months after that date. Following the official promulgation, a phase-in period will start that lasts a minimum of 2 years. After that period, all off-road construction machinery in Switzerland will have to be tested and certified for their PN emission biannually. A reference value for solid PN of 250,000 particles $/ \mathrm{cm}^{3}$ has been set as the pass/fail threshold under official measurement conditions. These conditions require a defined conditioning of engine and DPF first, so the measurement can be conducted at constant engine operation at maximum speed respectively high idle speed. The reference value chosen is analogue to the $1 \times 10^{12}$ particles $/ \mathrm{kWh}$ stated in the OAPC regulation.

\subsubsection{Nanoparticle Test System for the Swiss Ordinance on Exhaust Measurement}

The August 2012 and March 2014 amendments of the Ordinance of the FDJP on Exhaust Gas Analysers (VAMV) defined the specific requirements that nanoparticle measuring instruments must fulfill in Switzerland in order to test the corresponding types of construction machinery [32]. The main requirement is that such test systems have to be able to measure the number concentration of nanoparticles between 20 and $300 \mathrm{~nm}$ in diameter and between $5 \times 10^{4}$ and $5 \times 10^{6}$ particles $/ \mathrm{cm}^{3}$ in concentration. Similar to PMP, the volatile fraction of the emission is purposely excluded. The measurement must also be made with an efficiency of 70 to $130 \%$ for nanoparticles of $80 \mathrm{~nm}$ in diameter, which is a requirement that only CPCs meet perfectly. In addition, the test instrument 


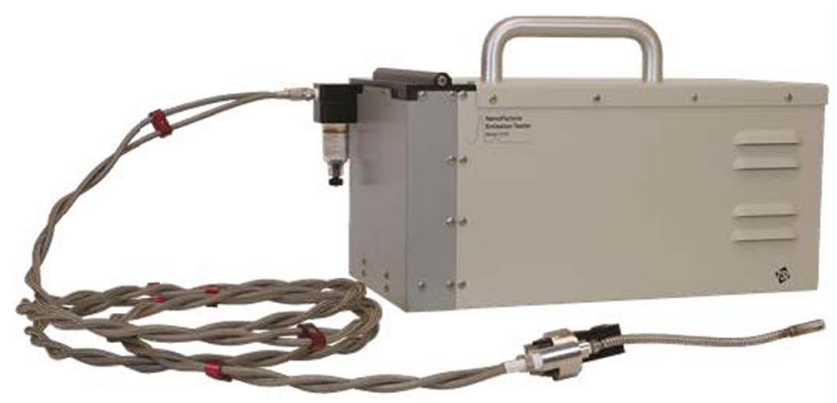

Fig. 3 Commercially available instrument for routine emissions testing of off-road machinery: nanoparticle emission tester (TSI model 3795, Photo (C) TSI, Inc.)

has to be configured for portable use and fulfill well-defined ambient temperature range and pressure conditions.

The first instrument that has been submitted for certification to the Swiss Federal Institute of Metrology (METAS) for official and routine emissions testing according to regulation SR 941.242 is the Nanoparticle Emission Tester (NPET) model 3795 (TSI Inc.) shown in Fig. 3. The NPET combines all of the commonly complex components in one portable instrument that can directly measure solid-particle number concentration in the range from at least 1,000 to 5,000,000 particles/ $\mathrm{cm}^{3}$ (Fig. 4). It consists of a stainless steel sample probe with a built-in 10:1 diluter that allows the measurement of high concentration emissions. Dilution air is provided internally by recirculating $6.3 \mathrm{~L} / \mathrm{min}$ of the sample flow after it passed through a diffusion dryer and two HEPA filters that dry and clean the air stream. Condensation of water vapor is prevented through drying the dilution air, in such a manner that the 10:1 dilution with dry air results in a mixed dew point greater than the coldest point in the measurement system. The diluted sample then enters the preconditioner, which consists of a combined $1-\mu \mathrm{m}$ cyclone to remove large particles and a water trap. This combination captures and eliminates any condensates that could be caused by cold environmental temperatures before they can enter the subsequent components of the instrument. Since the regulation once more defines that only the solid components of the exhaust emission shall be measured, a volatile particle remover is subsequently employed. It consists of a catalytic stripper $[1,14]$ that is heated to $350^{\circ} \mathrm{C}$ to remove greater than $99 \%$ of the volatile material from the sample flow. Any condensed hydrocarbon species in the sample are evaporated and oxidized by the catalytic stripper, whether they are emitted, formed during dilution, or caused by a storage and release event from the sampling system. The solid particles are then counted by a CPC that provides accurate measurements at high and low concentrations. This particular CPC uses isopropyl alcohol as its working fluid, which has been shown to be suitable for workplace environments both indoors and outdoors [16]. The NPET uses a built-in regulatory certification protocol that meets the Swiss regulation for the periodic testing of diesel engine exhaust aftertreatment systems but can also be operated in a more general mode of measurement.

Prototypes of additional test instruments for DPF inspection have been developed by University of Applied Sciences Northwestern Switzerland (FHNW) and Matter Aerosol AG, both of which use electrometers as their detector rather than CPCs. It should be noted that all of these instruments were specifically designed for field inspection and not test cell or chassis dynamometer environments. Although, they follow the essential ideas of PMP, they are not actually compliant with the PMP method.

\subsection{Aircraft Emission Legislation}

Aircraft engines have long been known to have a high particle number emission [28], a fact that until recently was not addressed by legislation. Instead the International Civil Aviation Organization (ICAO) relied on smoke number measurements introduced in the 1970s until very recently. In 2010, the ICAO's Committee on Aviation Environmental Protection (CAEP) expressed its interest in the development of a new methodology for measuring nonvolatile particle emissions. The focus on the nonvolatile fraction of the emission was explained by the scientific knowledge of solid particles being more advanced. The CAEP tasked the Society of Automotive Engineers (SAE) E-31 aircraft exhaust emissions measurement committee, which is a group of experts from industry, research, and regulators, with the development of a standardized measurement protocol. This protocol was then to be used to create an aerospace recommended practice for measuring
Fig. 4 Flow schematic of the nanoparticle emission tester (NPET), TSI model 3795

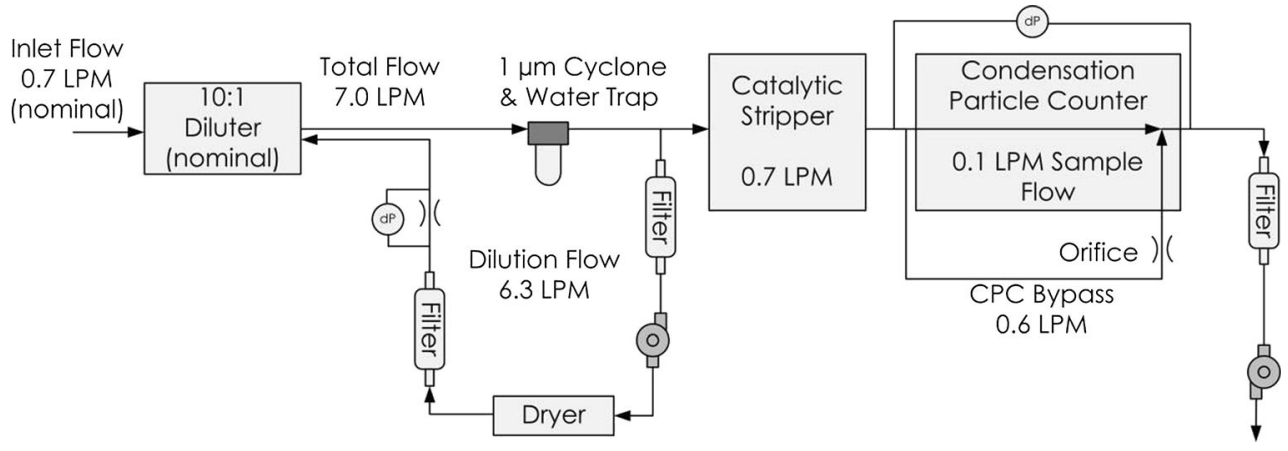


nonvolatile PM mass and particle number emissions from the exit plane of aircraft engines (SAE AIR).

A variety of aircraft and engines operated with different fuels have been tested during the SAMPLE, AAFEX, and APEX projects as well as a campaign at the SR Technics aircraft engine facility in December 2011 [24]. The testing was always done with a comprehensive suite of state-of-theart instrumentation to evaluate aircraft engines in terms of their gaseous compounds as well as particle size, number, and mass emissions. Following extensive consultation, the conclusion was reached to base a new measurement protocol for aircraft engine nonvolatile particulate matter (nvPM) and number emissions on the original method developed by PMP. An established regulation offers the advantage that it is well understood and already implemented for regulatory purposes. Although the PN emission method was adapted from PMP, it was modified to account for the different sampling needs and also to take into account that the size distribution of aircraft particle emissions is different from the one of on-road vehicles.

The SAE E-31 committee decided to focus on nonvolatile PN larger than $10 \mathrm{~nm}$ rather than the PMP's lower size limit of $23 \mathrm{~nm}$. This decision acknowledged that a greater share of the solid particle size distribution emitted by aircraft engines is in the sub-23-nm range than it is for on-road vehicles, while below $10 \mathrm{~nm}$ mainly volatile particles occur. According to Crayford et al. [4] more than $90 \%$ of the number of particles emitted by aircraft engines can be counted when using a 10$\mathrm{nm}$ lower detection limit. This modification to the test systems for measurements according to PMP can be accomplished with relative ease by exchanging the standard Engine Exhaust CPC typically used for PMP by one with a lower cutoff at $10 \mathrm{~nm}$, such as the model 3772 CPC (TSI Inc.).

The biggest challenge during the measurement of nvPM is to establish stable and reproducible sampling conditions. The
SAE E31 committee devised an elaborate sampling system for the simultaneous measurement of gases and particles that consists of three sections to address the collection, transfer, and measurement of the emission. This concept of the aircraft emission sampling system is presented in Fig. 5. As the schematic shows, much longer sampling lines with a total length of approximately $25 \mathrm{~m}$ are needed due to practical needs. The disadvantage of such a long sampling system is that it introduces significant particle losses predominately due to diffusion, so correction factors are needed to account for the line loss during both the particle number as well as the mass measurement.

The formulation of requirements for a nonvolatile particle number standard was achieved by the publication of the AIR 6241 document in November 2013. This aerospace recommended practice is expected to be the basis for the first nvPM standard for aircraft engine emissions that is presently developed by the CAEP working group for implementation in 2016.

\section{Future Emission Legislations for Particle Number}

With the aforementioned legislations that place mandatory limits on the PN emission, there is sufficient evidence of the day-to-day reliability, capacity, and usefulness of regulating solid particle number. Table 1 gives an overview of current and pending legislations that limit the particulate emission by their PN concentration.

It is expected that in the near future, the positive experience with PN measurement and the PMP method will expand to new sectors and additional emission sources will be targeted. One of the next steps will be diesel engines installed in nonroad mobile machinery (NRMM). NRMM is a broad category that covers equipment and vehicles used in road construction, railroad transport, marine vessels on inland waterways, as well

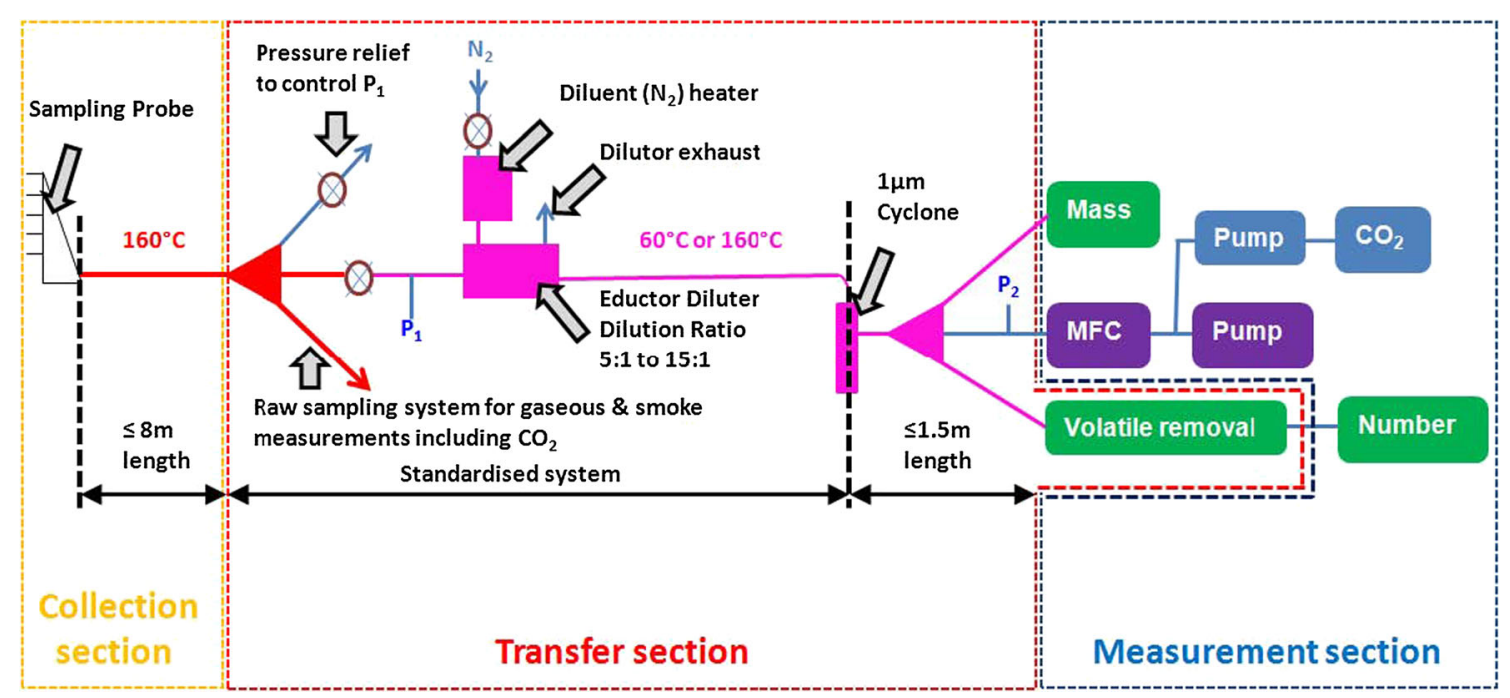

Fig. 5 Concept of the SAE E-31 sampling and measurement system for aircraft emissions [25] 
Table 1 European legislations that regulate particle number $(\mathrm{PN})$ emissions

\begin{tabular}{lllll}
\hline Vehicle category & Engine type & Standard & Introduction & PN limit \\
\hline Passenger cars & Light-duty diesel & Euro 5b & 2011 & $6 \times 10^{11} \mathrm{P} / \mathrm{km}$ \\
& & Euro 6a & 2014 & $6 \times 10^{11} \mathrm{P} / \mathrm{km}$ \\
& Gasoline direct injection & Euro 6b & 2014 & $6 \times 10^{12} \mathrm{P} / \mathrm{km}$ \\
& & Euro 6c & 2017 & $6 \times 10^{11} \mathrm{P} / \mathrm{km}$ \\
Trucks and buses & Heavy-duty diesel & Euro VI (WHTC) & 2013 & $6 \times 10^{11} \mathrm{P} / \mathrm{kWh}$ \\
& & Euro VI (WHSC) & 2013 & $8 \times 10^{11} \mathrm{P} / \mathrm{kWh}$ \\
Pending & & & & $1 \times 10^{12} \mathrm{P} / \mathrm{kWh}\left(2.5 \times 10^{5} \mathrm{P} / \mathrm{cm}^{3}\right)$ \\
Off-road construction machinery & Heavy-duty diesel & SR 941.242 & 2015 & $\mathrm{TBD}$ \\
Aircraft & Jet engine & (CAEP/10) & $(2016)$ & \\
\hline
\end{tabular}

as machinery used in the agricultural sector such as harvesters and tractors. In September 2014, the European Commission's Directorate-General for Enterprise and Industry met to discuss the revision of Directive 97/68/EC for NRMM. During that meeting, the proposal was made to adopt a PN emission limit for certification of select NRMM engine types that is to be enforced in January 2017. This proposal once again draws on the results of PMP by referring to the measurement of the number of solid particles larger than $23 \mathrm{~nm}$. Despite the fact that they also fall into the NRMM category, there is a related but currently separate activity for hand-held devices with internal combustion engines such as portable chainsaws.

The EU Commission is also working on developing legislation for real world emissions and has started to investigate $\mathrm{PN}$ emissions in that context. The corresponding measurement should be carried out during on-road vehicle tests with PN portable emission measurement systems (PN-PEMS). The motivation for such a regulation is to confirm that real driving emissions (RDE) match those measured during type approval certification under test cell conditions. A first assessment of portable commercial equipment for light-duty vehicles was presented by the European Commission's Joint Research Centre $[7,8]$. It is planned that a corresponding regulation will be proposed for light-duty diesel vehicles starting with Euro $6 \mathrm{c}$ in 2017, although no particle number limit has been set yet. Other sectors for the regulation of PN emissions include the large marine diesel engines used in cruise ships, tankers, and container ships, PN emissions from boilers and comparable heating systems as well as non-exhaust particulate emissions from vehicles such as brake and tire wear.

\section{Conclusions}

Advanced engine technology, modern fuels, new lubricants, and emission control devices have changed the nature of particulate emissions from most mobile sources. Emissions by on-road vehicles have been lowered significantly both qualitatively and quantitatively compared to barely a decade ago.
Despite the commendable advancements made, the measurement methods defined in legislation have long lagged behind that development. The conventional method of measuring particle mass (PM) consequently reached its limits and has been shown to be prone to artifacts at low emissions. Significantly more reliable results for low emissions can now be achieved by the much more sensitive solid particle number measurement method. While the PM mass method fails at very low emissions of $1 \mathrm{mg} / \mathrm{km}$ or less, the solid particle number method is so sensitive that it can measure emissions more than 20 times lower than that level. In addition, the measurement of PN offers the benefit that it can be used to indicate the correct performance of aftertreatment systems like DPFs or changes in their loading state.

The great advancement that was realized by the PMP program is that it developed and established a reproducible method to quantify very low particulate exhaust emissions. During the past few years, three emission legislations came into effect in Europe that made use of these findings and now limit the PN emission from on-road vehicles. PMP became one of the outstanding achievements for advancing emission standards in recent years, so that with regards to their particulate emissions, only the cleanest new vehicle types are approved to be used on European roads. At least two more PN legislations are well under way, the Swiss Ordinance on Exhaust Gas Analysers that takes PN measurements off-road, as well as the ICAO that applies it to the exhaust of aircraft turbine engines. Both are expected to come into effect within the next 2 years, with legislation for additional sources already being discussed. By reducing particulate emissions from this variety of mobile sources beyond the limit of detection of PM mass techniques, much can be accomplished to improve air quality.

It is necessary to point out that even bigger strides toward cleaner air could be made if PN emissions were not only regulated during type approval of new engines and vehicles. People living in the proximity of highly frequented roads and other sources of combustion emissions are frequently exposed to harmful levels of ultrafine particles. Such local air pollution and its severe adverse health impacts could be greatly curbed 
by the inclusion of PN concentration in ambient air quality standards. At present, there is no standard in place that regulates ambient levels of particle number anywhere in the world. The EU still has its regulatory focus on PM10 and PM2.5 mass concentrations while the number of ultrafines remains unregulated. In the USA, where regulatory authorities have reviewed the PMP method but not introduced a corresponding regulation for mobile sources, the US Environmental Protection Agency (EPA) has added ultrafines as an optional metrics to be measured in its near-road monitoring networks. In consequence, several US state and regional agencies entrusted with regulating air pollution have started adding instrumentation dedicated to the continuous monitoring of ultrafine particle number to their monitoring stations. This is a commendable effort that will enhance the understanding of the magnitude and nature of particulate emissions in the nanometer size range near busy roadways. It would be desirable for the EU to follow that example and implement a similar guidance.

Acknowledgments This paper is the result of a plenary lecture at the 1st APT-STEP workshop on Combustion Emission Control for Clean and Efficient Vehicles in Thessaloniki, Greece, which was funded by the European Union's Seventh Framework Programme under grant agreement no. 315871. I want to thank Dr. Athanasios Konstandopoulos and his team at the Aerosol and Particle Technology Laboratory of CERTH/ CEPRI for the kind invitation.

\section{References}

1. Abdul-Khalek, I., Kittelson, D.: Real time measurement of volatile and solid exhaust particles using a catalytic stripper, SAE Technical Paper 950236 (1995)

2. Andersson, J., Giechaskiel, B., Munoz-Bueno, R., Dilara, P. : Particle Measurement Programme (PMP): light-duty Inter-laboratory Correlation Exercise (ILCE LD)-final report (EUR 22775 EN), GRPE-54-08-Rev.1, http://www.unece.org/trans/main/wp29/ wp29wgs/wp29grpe/grpeinf54.html (2007)

3. Andersson, J., Mamakos, A., Giechaskiel, B., Carriero, M., Martini, G.: Particle Measurement Programme (PMP): heavy-duty Inter-laboratory Correlation Exercise (ILCE_HD) final report (EUR 24561 EN), Publications Office of the European Union, Luxembourg, ISBN 978-92-79-17200-7 (2010)

4. Crayford, A., Johnson, M.: Studying, sampling and measurement of aircraft particulate emissions II (SAMPLE. II) - final report EASA.2010.FC.10, European Aviation Safety Agency (2012)

5. Giechaskiel, B., Cresnoverh, M., Jörgl, H., Bergmann, A.: Calibration and accuracy of a particle number measurement system. Meas. Sci. Technol. 21, 045102 (2010)

6. Giechaskiel, B., Dilara, P., Andersson, J.: Particle measurement programme light-duty Inter-Laboratory Exercise: repeatability and reproducibility of the particle number method. Aerosol Sci Technol 42(7) (2008)

7. Giechaskiel, B., Martini, G.: Review on engine exhaust sub-23 nm particles. Publications Office of the European Union, Luxembourg, DRAFT 2014, accessed from: https://www2.unece.org/wiki/ download/attachments/16450001/GPRE-PMP-30-09\%
20DRAFT\%20Sub23nm\%20report_JRC_20140212.pdf?api=v2 (2014)

8. Giechaskiel, B., Riccobono, F., Bonnel, P. :Feasibility study on the extension of the real driving emissions (RDE) procedure to particle number (PN). Publications Office of the European Union, Luxembourg, JRC93743, ISBN 978-92-79-44650-4 (2014)

9. Giechaskiel, B., Wang, X., Horn, H.-G., Spielvogel, J., Gerhart, C., Southgate, J., Jing, L., Kasper, M., Drossinos, Y., Krasenbrink, A.: Calibration of condensation particle counters for legislated vehicle number emission measurements. Aerosol Sci. Technol. 43(12) (2009)

10. Kasper, M. : The number concentration of non-volatile particlesdesign study for an instrument according to the PMP recommendations. SAE Technical Paper 2004-01-0960 (2004)

11. Kasper, M.: Nanoparticle exhaust gas measurement: on-line response, high sensitivity, low cost. SAE Technical Paper 2003-010286 (2003)

12. Khalek, I., Bougher, T., Jetter, J.: Particle emissions from a 2009 gasoline direct injection engine using different commercially Available Fuels, SAE Technical Paper 2010-01-2117 (2010)

13. Kittelson, D.B.: Issue associated with solid particle measurements, 22nd CRC Real World Emissions Workshop, San Diego, CA, 2528 March, 2012 (2012)

14. Kittelson, D.B., Watts, W.F., Savstrom, J.C., Johnson, J.P.: Influence of a catalytic stripper on the response of real time aerosol instruments to diesel exhaust aerosol. J. Aerosol Sci. 36, 1089 1107 (2005)

15. Liu, W., Osmondson, B.L., Bischof, O.F., Sem, G.J.: Calibration of condensation particle counters. SAE Technical Paper 2005-010189 (2005)

16. Matson, U., Ekberg, L.E., Afshari, A.: Measurement of ultrafine particles: a comparison of two handheld condensation particle counters. Aerosol Sci. Technol. 38(5), 487-495 (2004)

17. Mayer, A., Czerwinski, J., Kasper, M., Ulrich, A., Mooney, J.: Metal oxide particle emissions from diesel and petrol engines, SAE Technical Paper 2012-01-0841 (2012)

18. Mayer, A., Czerwinski, J., Matter, U., Wyser, M., Scheidegger, P., Kieser, D., Weidhofer, J.: VERT: diesel nano-particulate emissions: properties and reduction strategies, SAE Technical Paper 980539 (1998)

19. Mohr, M., Lehmann, U.: Comparison study of particle measurement systems for future type approval application, Research report No-202779, Swiss contribution to GRPE particle measurement programme (GRPE-PMP CH5) (2003)

20. Peters, A., Wichmann, H.E., Tuch, T., Heinrich, J., Heyder, J.: Respiratory effects are associated with the number of ultrafine particles. Am. J. Respir. Crit. Care Med. 155(4), 1376-1383 (1997)

21. Regulation No. 49, E/ECE/324/Rev.1/Add.48/Rev.6, UNECE, (2013)

22. Regulation No. 83, E/ECE/324/Rev.1/Add.82/Rev.4, UNECE, (2011)

23. Reith, J., Horn, H.-G., Bischof, O.F., Krinke, Osmondson, B.: Features and advantages of a new engine exhaust condensation particle counter (EECPC), TSI Model 3790. $11^{\text {th }}$ ETH Conference on Combustion Generated Nanoparticles, Zürich, Switzerland, $14^{\text {th }}$ August 2007 (2007)

24. Rindlisbacher, T.: Non-volatile particle mass and number standard for aircraft gas turbines, $17^{\text {th }}$ ETH Conference on Combustion Generated Nanoparticles, Zürich, Switzerland, 24 ${ }^{\text {th }}$ June 2013 (2013)

25. SAE AIR6241 Procedure for the continuous sampling and measurement of non-volatile particle emissions from aircraft turbine engines, aerospace information report, SAE International (2013)

26. Schlatter, J.: Diesel smoke measurements — optical methods versus particle counting. $12^{\text {th }}$ ETH Conference on Combustion Generated Nanoparticles, Zürich, Switzerland, $23^{\text {rd }}$ June 2008 (2008) 
27. Schlatter, J.: New Swiss legislation on portable particle counters for construction machinery. $16^{\text {th }}$ ETH Conference on Combustion Generated Nanoparticles, Zürich, Switzerland, 24 ${ }^{\text {th }}$ June 2012 (2012)

28. Schröder, F.P., Kärcher, B., Petzold, A., Baumann, R., Busen, R., Hoell, C., Schumann, U.: Ultrafine aerosol particles in aircraft plumes: in situ observations. Geophys. Res. Lett. 25(15), 27892792 (1998)

29. Sem, G.J.: Design and performance characteristics of three continuous-flow condensation particle counters: a summary. Atmos. Res. 62, 267-294 (2002)

30. Sem, G.J., Bischof, O.F., Kittelson, D.B.: Review of particle size distribution measurements of engine exhaust before 1985. In: Ensor, D.S. (ed) Aerosol science and technology: history and reviews. RTI Press, Research Triangle Park, NC (2010)

31. Spiegel, J., Andres, H., Hogstrom, R., Jordan-Gerkens, A., Nowak, A.: Laboratory evaluation of novel soot sensors for periodic emission control of modern diesel vehicles, ENV02 PartEmission/WP2/ Task 2.1/Deliverable2.1.2 (2014)
32. SR 941.242, Ordinance of the FDJP on Exhaust Gas Analysers (VAMV), Amendment No. II on August 22, (AS 2012 5371) (2012)

33. TRGS 900, Technische Regel für Gefahrstoffe, Grenzwerte in der Luft am Arbeitsplatz "Luftgrenzwerte", GMBI (1996)

34. US Patent 7,682,426 B2. Method and device for the detection, characterization and/or elimination of suspended particles. Issued March 23, (2010)

35. Wei, Q., Akard, M., Asano, I., Rahman, M.: Penetration, calibration and verification for the solid particle counting system with polydisperse and monodisperse particles. SAE Int. J. Fuels Lubricants 1(1), 593-602 (2009)

36. Wei, Q., Ichiro, A., Adachi, M., Montajir, R., Kusaka, T., Goto, Y. Real-time measuring system for engine exhaust solid particle number emission - performance and vehicle tests. SAE Technical Paper 2006-01-0865 (2006)

37. Zheng, Z., Johnson, K.C., Liu, Z., Durbin, T.D., Hu, S., Huai, T., Kittelson, D.B., Jung, H.S.: Investigation of solid particle number measurement: existence and nature of sub-23nm particles under PMP methodology. J. Aerosol Sci. 42(12), 883-897 (2011) 